\title{
CHANGE DETECTION-BASED UPDATING OF CONSTRUCTED LAND IN LARGE AREA
}

\author{
H. Q. Xing ${ }^{\mathrm{a}, \mathrm{b}^{*}}$, J. Chen ${ }^{\mathrm{b}}, \mathrm{H} . \mathrm{Wu}^{\mathrm{b}}, \mathrm{M} . \mathrm{Lu}^{\mathrm{b}, \mathrm{c}}$
${ }^{a}$ Geoscience and Surveying Engineering College, China University of Mining and Technology(Beijing), Beijing,100083, China -xinghuaqiao@126.com
${ }^{\mathrm{b}}$ National Geomatics Center of China, 28 Lianhuachi West Road, Beijing 100830, China
${ }^{\mathrm{c}}$ School of Remote Sensing and Information Engineering, Wuhan University, Wuhan 430079, China

KEY WORDS: Land cover updating, Change detection, Large area

\begin{abstract}
:
Urban development significantly changes constructed land across both urban and rural areas around the world in recent years. It is vital to keep constructed land data "fresh" for its application and development. Change detection with remotely sensed imagery is an effective way to updating land cover in large area. In this paper, the updating requirements of constructed land in large area are analysed. A strategy is proposed to select suitable change detection method from data source, manual work and accuracy assessment. Then two different approaches of updating land cover with change detection are compared by mean of this strategy. Based on the comparison result, current experiment of updating constructed land with change detection in Shandong province is discussed. Updating land cover with change detection in manner of web services will be research priorities in future research.
\end{abstract}

\section{INTRODUCTION}

Global land cover (GLC) data is fundamental for many application areas, especially for climate change and global sustainability studies (Foley J., et al., 2005; Feranec, J., et al., 2007; Chen et al., 2011).As one type of land cover, constructed land changes a lot because of urban development in recent years. Continuous, accurate, and up-to-date land cover data are important for natural resource and ecosystem management and need to be based on consistent monitoring of landscape attributes over time (Xian et al., 2009; Jin et al., 2013). It is vital to keep constructed land data "fresh" for its application and development. If constructed land data cannot be updated timely, the correct analysis results will not be provided. The GLC product of 2010 (GLC 2010) may not meet user community latest needs. For instance, some country or district wants to use constructed land data or information in 2013, while GLC 2010 cannot reflect the correct land cover types in 2013. Therefore, it is imperative to update constructed land data to the latest one.

To achieve this goal in a cost-effective manner, one possible approach would be to identify areas of LC change occurring subsequent to 2010 and to update LC data only for these areas (Xian et al., 2009; Hulley, G., et al., 2014).. For the areas that have not changed, the GLC 2010 land cover would remain the same. Land cover updating change detection with remotely sensed imagery is one of the effective ways, in which the change area is detected by comparing and analysing images of base year and detective year, and the types of the changed area will be updated to the latest ones.

In recent decades, some organizations and institutes have made certain progress on land cover updating. The U.S. Geological Survey (USGS) update the 2001 National Land Cover Database land cover classification to 2006 by using Landsat imagery change detection methods (Xian et al., 2009). To continue providing updated national land cover and change datasets, they present a new Comprehensive Change Detection Method (CCDM) designed as a key component for the development of NLCD 2011 and the research results from two exemplar studies (Jin et al., 2013), in this method, four spectral indices (CV, RCVMA X, dNBR, and dNDVI) to obtain the changes that occurred between two image dates. It can capture a variety of natural and anthropogenic disturbances potentially associated with land cover changes on different landscapes. From the point of view of land cover updating automation, an automated approach for updating land cover maps is proposed which based on integrated change detection and classification methods (Chen et al., 2012), in this proposed approach, remotely sensed data acquired in different seasons or from different remote sensors can be used. In order to decrease pseudo changes detected by change detection, a new method is proposed which is called Spectral Gradient Difference (SGD) (Chen et al., 2013); SGD can quantitatively describe the spectral shapes and the differences in shape between two spectra, change magnitude calculated in the new spectral gradient space is used to detect the change/no-change areas.

However, land cover updating with change detection in large area face a lot of challenges, the selection of land cover change detection method and updating technique process is the largest one. To begin with, there are different features associated with different change detection methods. Most change detection methods have its suitable data and area condition, there are no method can be suitable for all data and area condition in large area. Moreover, land data updating with imagery change detection is a complicated technique process, which includes many processes such as imagery preprocessing, imagery change detection, imagery classification and data integration. In the process of land data updating, each process has many models or algorithms, not all image data need the same technique process of land data updating or the same models or algorithms. Traditionally, the existing researches on land cover change detection often focused on small area, and are only suitable for one type of data source, in addition, the technological processes of land cover data updating are offline operations, which are often appointed by professional manually, and much software like ENVI, ERDAS is needed installed. Without these software and algorithms, it is difficult to update land cover data by users 
form any other areas. Therefore, the models or algorithms of land cover change detection for updating are crying needed sharing on the internet, and then users can choose suitable models or algorithms of land cover change detection for updating in the manner of web services.

In light of the above mentioned problems, the tasks of this paper focus on the follow two aspects. On the one hand, how to select the suitable change detection algorithms and updating technique process in land cover updating of large area. On the other hand, how to support web service-based land cover change detection using the knowledge mentioned above. The structure of this paper is as follows. Two approached of updating land cover with change detection are discussed and compared in section 2, each of them has its advantages and disadvantages, and we propose a method to select the suitable algorithm and updating technique process according to data source and area condition in large area. In section 3 and 4, experiment in a case of Shandong province is conducted to validate our proposed method. Finally, conclusion and future work are presented in section 5 .

\section{A STRATEGY TO SELECT SUITABLE CHANGE DETECTION METHOD}

From different points of view, land cover change detection methods can be categorized into direct radiometric comparison methods, post-classification methods, object oriented change detection, time series methods and so on. These methods often have specific requirement of data source, detection object and environment, there have no method can be suitable for all application; the conclusions drawn from different methods sometimes are even diverse. Consequently, it is quite important to summarize the applicability of land cover change detection for updating. From analysing land cover change detection methods, the characters of the methods can be drawn: diversity of methods, complexity of process and versatility of accuracy assessment. The reasons of these characters may be caused by diversity of remote sensing imagery, manual work in the process and many index of accuracy assessment. There are different imagery data such as Landsat data, MODIS data, HJ-1A data, SPOT data, QuickBird data and so on, which are different in their spatial resolution, spectral resolution, radiation resolution and temporal resolution. Different change detection methods have different requirement of resolutions consistency of imagery pairs. For instance, radiometric comparison methods require imagery pairs of the same spectral resolution and radiation resolution, while for post-classification methods, the imagery pairs of the same spectral resolution and radiation resolution can be different. In addition, change detection methods are different in the extent of manual work; some methods need much manual work in training samples selection or threshold selection, while some methods need less one. Accuracy of change detection methods can be assessed in overall accuracy, Kappa coefficient, commission error, and omission error, some methods may be lower in commission error, while some methods may be lower in omission error. Therefore, we should firstly summarize the applicability of land cover change detection for updating from diversity of data source, extent of manual work and different index of accuracy assessment.

In this paper, we select and compare two different change detection methods: a spectral gradient difference (SGD) based approach for land cover change detection (Chen et al., 2013) and an automated approach for updating land cover maps based on integrated change detection and classification methods (Method
B) (Chen et al., 2012). From analysing the applicability of two methods, SGD belongs to direct radiometric comparison methods, it use spectral gradient difference to quantitatively describe the spectral shapes and the differences in shape between two spectra, and change magnitude calculated in the new spectral gradient space is used to detect the change/nochange areas, and then a chain model is employed to represent the SGD pattern both qualitatively and quantitatively (Figure 1). SGD requires high consistency of spectral resolution and radiation resolution between two different images, and it also requires much manual woks in change threshold selection and sample selection for land cover updating.

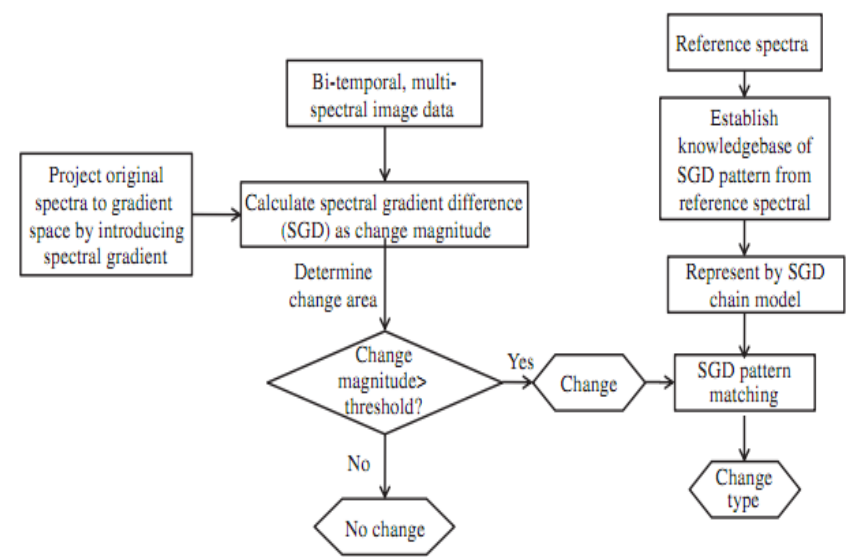

Figure 1.Framework of SGD based change detection (Chen et al., 2013)

An automated approach for updating land cover maps based on integrated change detection and classification methods belongs to post-classification methods. This method consists primarily of the following three parts: a change detection technique, a Markov Random Fields (MRFs) model, and an iterated training sample selecting procedure. In the proposed approach, remotely sensed data acquired in different seasons or from different remote sensors can be used. Meanwhile, the approach is completely unsupervised. The flowchart of this method is shown in Figure 2.

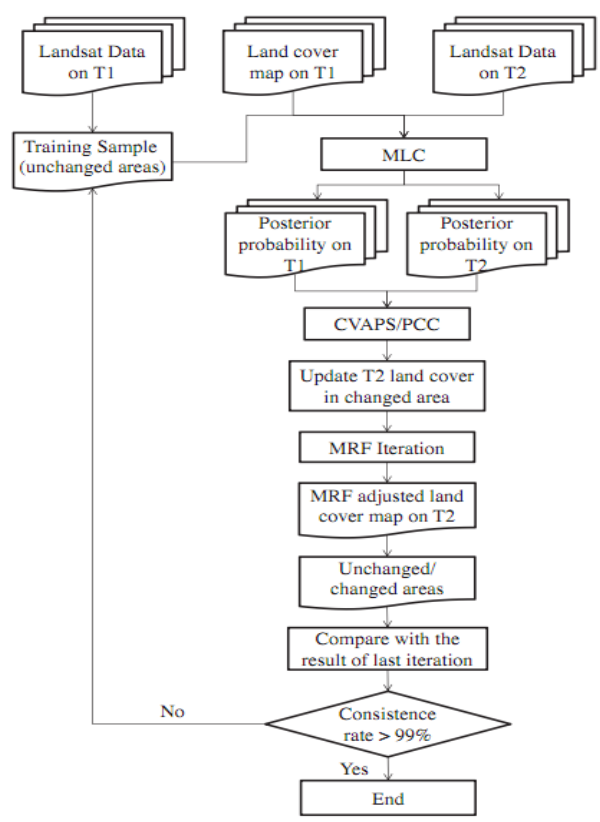

Figure 2.Flowchart of the proposed approach (Chen et al., 2012). 
In this experiment, two time image data are Landsat5 TM in 2010 and Landsat8 TM in2013, the radiation resolution of them is 8bit and 16bit, and the spectral resolution is also different(7 bands and 9 bands). For another consideration, this experiment is in large scale, it will need much manual work if we choose SGD, so we choose method B even if accuracy of SGD is a little higher than method $\mathrm{B}$.

\section{STUDY AREA AND DATA}

\subsection{Shandong province}

In this paper, we select Shandong province as study area, and update constructed land to 2013. Shandong province is located in east longitude $114^{\circ} 19^{\prime}-122^{\circ} 43^{\prime}$, and north latitude $34^{\circ} 22^{\prime}-38^{\circ} 23^{\prime}$. Constructed land in Shandong province especially in country and town level change a lot because of rapid development of economy. The importance of industrial structure and urbanization interaction has been proved by our country practice since reform and opening. Urbanization and industry of Shandong province is the most important factor which prompts the change of constructed land. A lot of buildings and factories are built on cropland and other land cover type. As discussed above, GLC 2010 cannot reflect correct land cover types in 2013. Therefore, urban planning of Shandong province urgently wants to update impervious area of GLC data 2010 to 2013 in county and district level.

\subsection{Land cover data}

GLC 2010 product at 30m resolution has been finished completely by china. GLC 2010 has 10 land cover types include water bodies, impervious area, wetland, croplands, forests, grasslands, shrublands, tundra, barren land and permanent snow/ice, which has been published in the manner of data web services. (Figure3). Users can visit GLC website named "Higher Resolution Global Land Cover Mapping" to view the GLC product in 2000 and 2010 (URL:http://www.globallandcovere.com).GLC 2010 product is organized by map sheets. Shandong province has 3 map sheets; the map sheet number is N50_35, N51_35, and N50_30.
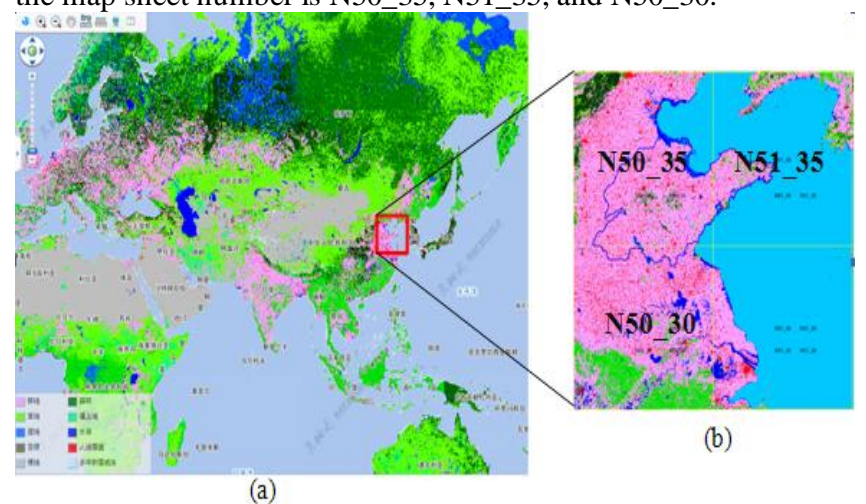

Figure 3. GLC 2010 at 30m resolution (a) and land cover product in Shandong province (b)

\subsection{Landsat data}

Images data selected in this experiment include Landsat8 TM in 2013, Landsat5 TM in 2010, which have 14 pairs of Landsat data (Figure 4); the WRS path/row number is: p123r034、p123r035、p123r036、p122r034、p122r035、 p122r036、p121r034、p121r035、p121r036、p120r034、 p120r035、p120r036、p119r034、p119r035, respectively.

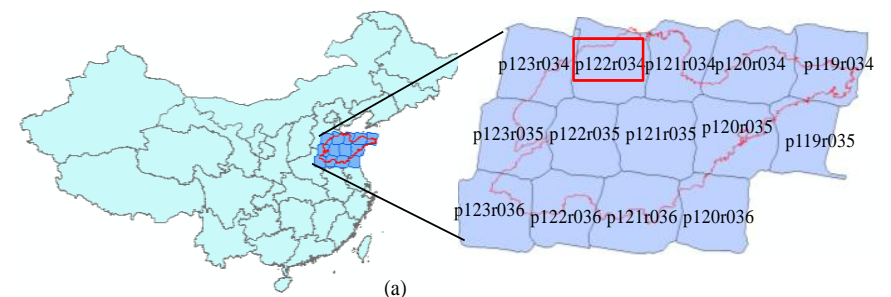

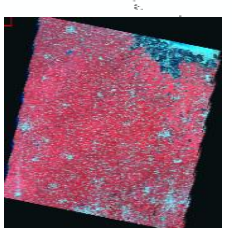

(b)

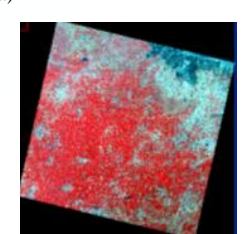

(c)
Figure 4.Study area (a) and Landsat imagery of p122r034: Landsat5 TM (b), Landsat8 TM (c)

\section{EXPERIMENT}

Image preprocessing is a necessary step for updating. Therefore, in the first step, the image-to-image geometric registration was conducted between the Landsat images and GLC 2010. The registration error was less than 0.5 pixels. In addition, the projection information and spatial scope of Landsat images and GLC 2010 must be consistent (Figure 5).
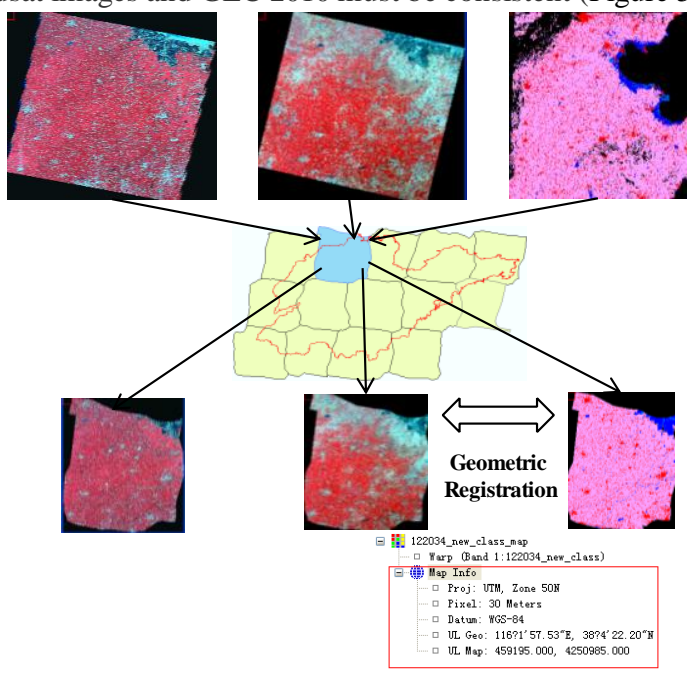

Figure 5.Data preprocessing

In this experiment, residential area above town and county level is primary area for updating. 1:1,000,000 Residential point data (include town, country, city and province), Google Earth image and constructed land of GLC 2000 are used for explicit the updating area, which can improve updating efficiency (Figure 6). 


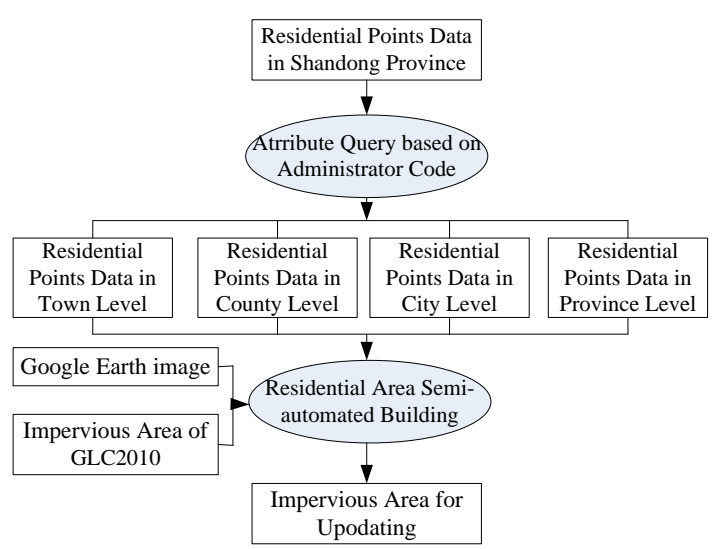

Figure 6.Updating area of Shandong province

As discussed in section 2, from methods applicability of diversity of data source, extent of manual work and different index of accuracy assessment, an automated approach for updating land cover maps based on integrated change detection and classification methods is suitable for our experiment. By the automated method, land cover in Shandong province is update to 2013 scene by scene (Figure 7). Impervious area can be extracted from the latest land cover. Finally, entire impervious area of Shandong province is joined together by mosaic function of ENVI (Figure 8).

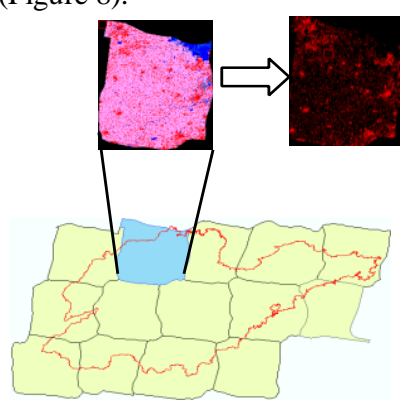

Figure 7. Constructed land updating scene by scene

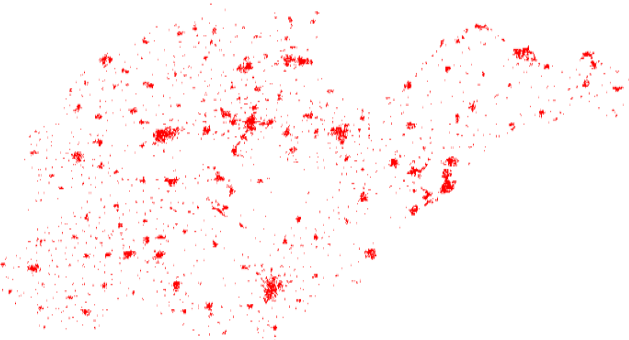

Figure 8. Constructed land 2013 of Shandong province (red area)

\section{CONCLUSION AND FUTURE RESEARCH}

Change detection with imagery is an effective method for land cover updating in large area, in which the change area is detected by comparing and analysing images of base year and detective year, and the types of the changed area will be updated to the latest ones while the types of unchanged area will be remained. There are a lot of problems in change detection for updating in large area like algorithms and updating technique process selection. In this paper, we compare two different approaches of updating land cover with change detection, and analysis their suitable data source and updating flow. At last, an experiment of updating constructed land in Shandong province is conducted to validate the idea we proposed.

As discussed above, Change detection-based updating of land cover in large area face a lot of challenges such as algorithms and updating flow selection, Collaborative updating.
Web-based change detection for updating may be an effective way to updating land cover in large area. Therefore, future research work will be conducted on several aspects:

1. Build knowledge model of land cover updating based on applicability of land cover change detection for updating;

2. Describe web processing service with applicability of land cover change detection, on this base, mining web service relation.

3. Web processing service dynamic composition based on service relation of expression and reasoning.

\section{REFERENCE}

Chen, J., Chen J., Gong P., 2011. Higher Resolution Global Land Cover Mapping. GEOMATICS WORLD, 12-14.

Chen, J., Gong, P., He, C., Pu, R., Shi, P., 2003. Land-use/landcover change detection using improved change-vector analysis. Photogrammetric Engineering \&Remote Sensing 69 (4), 369 379.

Chen, X., Chen, J., Shi, Y., Yamaguchi, Y., 2012. An automated approach for updating land cover maps based on integrated change detection and classification methods. ISPRS Journal of Photogrammetry and Remote Sensing 71 (7), 86-95.

Chen, J., et al. Temporal logic and operation relations based knowledge representation for land cover change web services. ISPRS J. Photogram. Remote Sensing (2013), http://dx.doi.org/10.1016/j.isprsjprs.2013.02.005.

Chen, J., Lu, M., Chen X., 2013. A spectral gradient difference based approach for land cover change detection. ISPRS Journal of Photogrammetry and Remote Sensing 85 (2013) 1-12.

Feranec, J., Hazeu, G., Christensen., S., 2007. Corine land cover change detection in Europe (case studies of the Netherlands and Slovakia). Land Use Policy 24 (2007) 234-247.

Foley J., et al. Global consequences of land use [J]. Science, 2005, 309:570-574.

Hansen, M., Loveland, T., 2012. A review of large area monitoring of land cover change using Landsat data. Remote Sensing of Environment, 122, 68-74.

Hulley, G., Veraverbeke, G., Hook, S., 2014. Thermal-based techniques for land cover change detection using a new dynamic MODIS multispectral emissivity product (MOD21). Remote Sensing of Environment 140 (2014) 755-765.

Jin, S., Yang, L., Danielson, P., 2013. A comprehensive change detection method for updating the National Land Cover Database to circa 2011. Remote Sensing of Environment, 132, 159-175.

Li, D., 2010. Remotely sensed images and GIS data fusion for automatic change detection. International Journal of Image and Data Fusion 1 (1), 99-108.

Lucas, R., Medcalf, K., Brown, A., 2011. Updating the Phase 1 habitat map of Wales, UK, using satellite sensor data. ISPRS Journal of Photogrammetry and Remote Sensing 66 (2011) 81102.

Were, K., Dick, Ø., Singh, B., 2013. Remotely sensing the spatial and temporal land cover changes in Eastern Mau forest reserve and Lake Nakuru drainage basin, Kenya. Applied Geography 41 (2013) 75-86.

Xian, G., \& Homer, C., 2009. Updating the 2001 National Land Cover Database impervious surface products to 2006 using Landsat imagery change detection methods. Remote Sensing of Environment, 114, 1676-1686.

Xian, G., Collin, H., Fry, J., 2009. Updating the 2001 National Land Cover Database land cover classification to 2006 by using Landsat imagery change detection methods. Remote Sensing of Environment 113 (6), 1133-1147. 